\title{
Erratum to: Temporal characterization of serum metabolite signatures in lung cancer patients undergoing treatment
}

\author{
Desirée Hao $^{1}$ - M. Omair Sarfaraz ${ }^{2,3}$ - Farshad Farshidfar ${ }^{4}$ D. Gwyn Bebb ${ }^{1}$. \\ Camelia Y. Lee ${ }^{5}$ Cynthia M. Card ${ }^{1} \cdot$ Marilyn David $^{6} \cdot$ Aalim M. Weljie $^{2,7,8}$ (D)
}

Published online: 2 July 2016

(c) Springer Science+Business Media New York 2016

\section{Erratum to: Metabolomics (2016) 12:58 \\ DOI 10.1007/s11306-016-0961-5}

The original version of this article unfortunately contains a mistake. The presentation of Fig. 2e and its caption are incorrect. The corrected Fig. 2e and its caption are given below.

The online version of the original article can be found under doi:10.1007/s11306-016-0961-5.

Aalim M. Weljie

aalim@upenn.edu

1 Department of Medical Oncology, Tom Baker Cancer Centre and Cumming School of Medicine, University of Calgary, 1331-29th Street N.W., Calgary, AB T2N 4N2, Canada

2 Department of Biological Sciences, University of Calgary, Calgary, AB T2N 1N4, Canada

3 Department of Medicine-Pathology and Molecular Medicine, McMaster University, Hamilton, ON L8N 3Z5, Canada

4 Department of Medical Oncology, Cumming School of Medicine, University of Calgary, 1331-29th Street N.W., Calgary, AB T2N 4N2, Canada

5 Tom Baker Cancer Centre, 1331-29th Street N.W., Calgary, AB T2N 4N2, Canada

6 Clinical Research Unit, Tom Baker Cancer Centre, 1331-29th Street N.W., Calgary, AB T2N 4N2, Canada

7 Institute of Translational Medicine and Therapeutics, University of Pennsylvania, Philadelphia, PA 19104, USA

8 Department of Systems Pharmacology and Translational Therapeutics, Perelman School of Medicine, University of Pennsylvania, Philadelphia, PA 19104, USA 

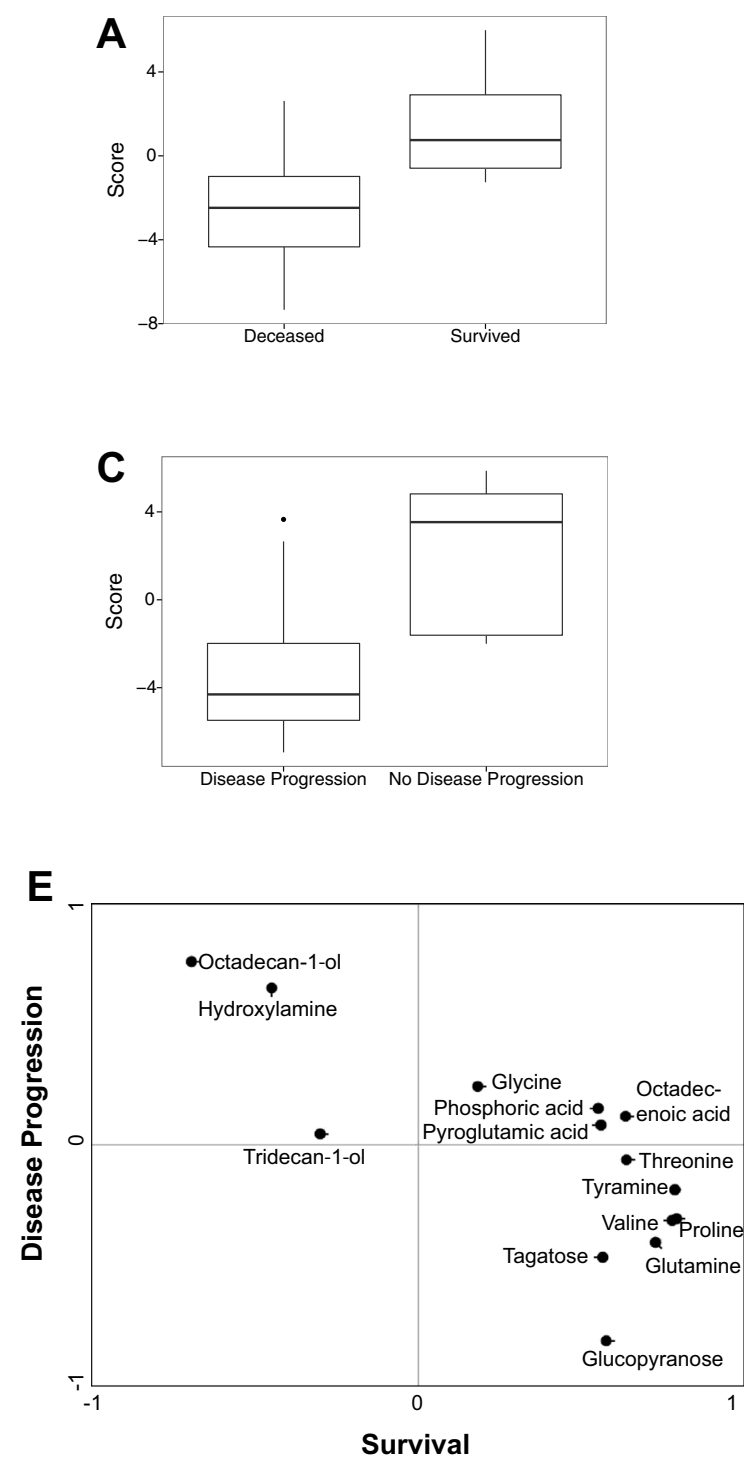

Fig. 2 GC-MS metabolite bioprofiling facilitates prognostic evaluation of clinical outcomes based on survival and disease progression. a Box and whisker plot based on scores from OPLS-DA model of patient survival at pretreatment as a function of the eventual survival status: b Heat map showing clustering of metabolites with respect to patient survival (c, d) Progression: $\mathbf{c}$ as in a, with samples stratified by

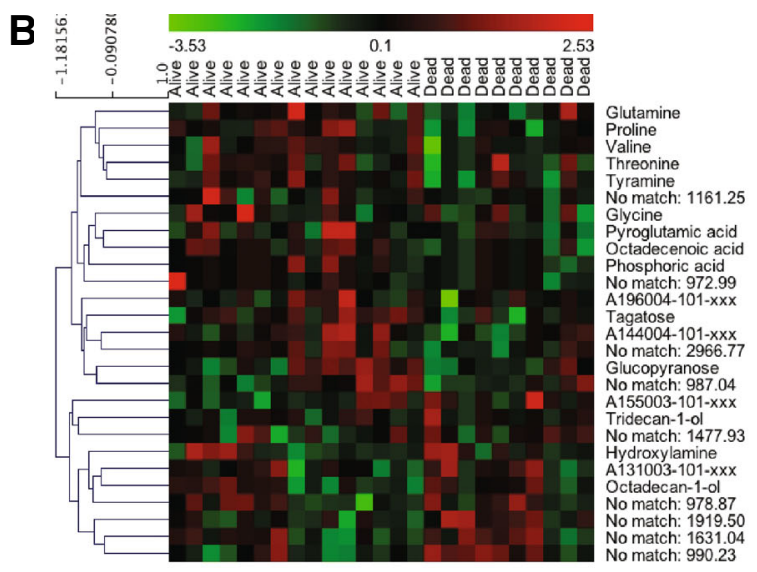

D
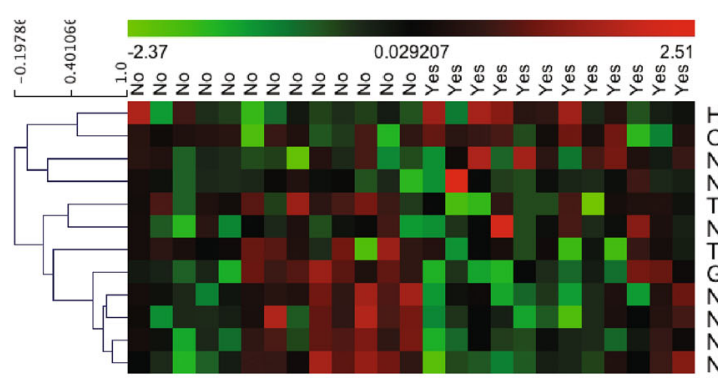

Hydroxylamine Octadecan-1-ol No match: 1919.50 No match: 972.99 Tagatose No match: 1161.25 Threonic acid Glucopyranose No match: 1045.20 No match: 1164.06 No match: 987.04 evidence of progression; d Heatmap showing progression-related metabolites; e Shared and unique structure (SUS) Plot, highlighting the strong relation between the two variables of disease progression and survival. The metabolites that line up along the diagonal running from the upper left corner to the lower right corner are common to both the disease progression and survival models 